\title{
Long-Term Clinical Course of Benign Fibro-Osseous Lesions in the Paranasal Sinuses
}

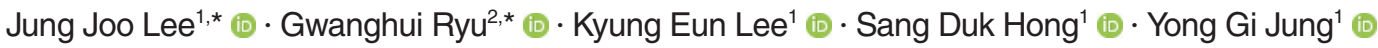 \\ Hyo Yeol Kim ${ }^{1}$ (D) Hun-Jong Dhong ${ }^{3}$ (i) $\cdot$ Seung-Kyu Chung ${ }^{1}$ [D \\ ${ }^{\prime}$ Department of Otorhinolaryngology-Head and Neck Surgery, Samsung Medical Center, Sungkyunkwan University School of Medicine, Seoul; \\ ${ }^{2}$ Department of Otorhinolaryngology-Head and Neck Surgery, Soonchunhyang University College of Medicine, Cheonan;
}

${ }^{3}$ Department of Rhinology, Hana ENT Hospital, Seoul, Korea

Objectives. Fibro-osseous lesions of the paranasal sinuses can present various clinical manifestations. This study aimed to report the long-term clinical course of benign fibro-osseous lesions (BFOLs) in the paranasal sinuses, including clinical and radiologic features.

Methods. Radiologically confirmed BFOLs between 1994 and 2016, with the exclusion of osteoma cases, were retrospectively reviewed. We compared demographic characteristics between the surgery and observation groups. The reasons for the imaging study, radiographic features, histopathology, and clinical course based on serial image scans were analyzed.

Results. In total, 183 subjects were selected from a thorough review of head and neck radiologic tests $(n=606,068)$ at a tertiary referral hospital over 22 years. Patients' mean age was $28.6 \pm 18.1$ years, and $56.3 \%$ were males. A diagnostic imaging workup was performed in $55.7 \%$ of patients due to facial asymmetry, headache, skull mass, or other symptoms related to BFOLs. In other patients (37.7\%), BFOLs were found incidentally on computed tomography or magnetic resonance imaging. The most common diagnosis was fibrous dysplasia, followed by ossifying fibroma, based on both radiologic exams and histopathologic results. In total, $42.6 \%$ of the patients underwent surgery because of subjective symptoms or esthetic concerns. The patients who underwent surgery were younger $(P<0.001)$ and had a longer follow-up duration $(P<0.001)$ than those who underwent observation. Patients who experienced lesion growth $(11.5 \%)$ were younger $(P<0.001)$ and had more lesion sites $(P=0.018)$ than those who did not, regardless of surgical treatment. Five patients underwent optic nerve decompression, and one patient experienced malignant transformation.

Conclusion. BFOL in the paranasal sinuses is a rare disease, and most cases were observed without specific treatment. Surgical treatment should be considered in symptomatic patients with aggressive clinical features. Regular observation and management are needed, particularly in younger patients in their teens.

Keywords. Fibro-Osseous Lesion; Paranasal Sinus; Fibrous Dysplasia; Ossifying Fibroma

\footnotetext{
- Received July 17, 2020

Revised October 5, 2020

Accepted October 29, 2020

- Corresponding author: Seung-Kyu Chung

Department of Otorhinolaryngology-Head and Neck Surgery, Samsung

Medical Center, Sungkyunkwan University School of Medicine,

81 Irwon-ro, Gangnam-gu, Seoul 06351, Korea

Tel: +82-2-3410-3579, Fax: +82-2-3410-3879

E-mail: rhinochung@gmail.com

*These authors contributed equally to this work.
}

\section{INTRODUCTION}

Benign fibro-osseous lesions (BFOLs) of the paranasal sinuses are variations along the intraosseous disease spectrum that exhibit similar histologic characteristics: hypercellular fibroblastic stroma containing various combinations of bone or cementumlike tissue and other calcified structures [1]. BFOLs have osseous and fibrous tissue components. Variation in terms of the fibrous element encompasses monomorphic fibroblasts and hy-

Copyright (C) 2021 by Korean Society of Otorhinolaryngology-Head and Neck Surgery.

This is an open-access article distributed under the terms of the Creative Commons Attribution Non-Commercial License (https://creativecommons.org/licenses/by-nc/4.0)

which permits unrestricted non-commercial use, distribution, and reproduction in any medium, provided the original work is properly cited. 
percellular thin collagen fibers. Their ossification-related features are also quite variable, including metaplastic woven bone, osteoblastic rimming, trabecular paralleling, and curvilinear conglomerates [2]. Osteoma, fibrous dysplasia (FD), and ossifying fibroma $(\mathrm{OF})$ are the main BFOLs of the paranasal sinuses. FD is a dysplastic skeletal anomaly characterized by the replacement of normal bone with immature bone and fibrous tissue. It may affect a single bone (monostotic) or multiple bones (polyostotic). Polyostotic FD is less common, and is sometimes related to McCune-Albright syndrome, which also involves skin pigmentation and endocrine abnormalities [3]. OF is rare and typically found in the mandible; therefore, it is usually reported by oral surgeons [4].

The histologic diagnosis of BFOL is quite challenging for the pathologist, and an accurate diagnosis in most patients therefore requires a comprehensive assessment of histologic, clinical, and radiologic findings [5]. Usually, the first diagnostic step is a radiologic imaging test. However, because the ossifications and fibrous components of BFOLs can be quite heterogeneous even within specific disease entities, it is difficult to reach an accurate diagnosis based only on imaging, except for simple osteomas [6]. The choice of treatment should be individualized in each case based on clinical symptoms and the location of paranasal fibroosseous lesions. The choice of whether to perform surgical treatment of these lesions depends on subjective symptoms or esthetic concerns. In the absence of symptoms, slow-growing lesions can often be managed conservatively through observation. Surgery is the most effective therapeutic strategy, particularly for lesions involving the orbit or the skull base [7].

Because BFOLs of the paranasal sinuses are rare, their appropriate management has not been well established. A small number of studies have reported the clinical manifestations and treatment outcomes of BFOL. This study had two purposes. First, we aimed to identify different types of BFOLs involving the paranasal sinuses in a large study population and to analyze their clinical and radiologic characteristics. Second, we tested the hypothesis that some clinical factors would be related to the recurrence of BFOLs after surgical management. Therefore, we reviewed the long-term follow-up results of BFOL patients.

\section{H I G H L I I G H T S}

- Benign fibro-osseous lesions (BFOLs) in the paranasal sinuses are rare, and most cases are observed without specific treatment.

- Surgical treatment should be considered in symptomatic patients with aggressive clinical features.

- A multidisciplinary approach is needed for accurate diagnosis and appropriate management of BFOLs of the paranasal sinuses, particularly in younger patients in their teens.

\section{MATERIALS AND METHODS}

\section{Study population}

We pulled out a list of head and neck imaging tests, including computed tomography (CT) and magnetic resonance image from October 1994 to January 2016 in a tertiary referral hospital. We searched for cases marked FD or OF on the official image reports and reviewed the image of those patients. Bony tumors confined to the paranasal sinuses were defined as BFOLs according to the diagnosis of a radiologist. Osteomas were excluded except for osteoma as a differential diagnosis of FD or OF. The reason for the exception is that osteoma is often diagnosed only by CT scan, and many cases are not treated if patients have no symptoms. The CT findings of FD were expansile, ground glass, sclerotic, or lytic lesions [8]. The radiologic features of OF were described as round lesions with sclerotic eggshell rim and central heterogeneity [6]. We retrospectively reviewed medical records of patients with radiologically diagnosed BFOL (FD or OF) of the paranasal sinuses. After a thorough imaging review, cases with BFOL in the craniofacial area that did not involve the paranasal sinuses were excluded. We analyzed data, including age, sex, clinical manifestations, sites of involvement, the reason for imaging, radiologic results, surgical histology, and clinical course with serial image scans. This study was approved by the Institutional Review Board of Samsung Medical Center (IRB No. 2019-01-105), and the need for informed consent was waived.

\section{Evaluation of management modalities}

If the patients had no symptoms and lesions were slow-growing on follow-up imaging, they were followed regularly at an outpatient clinic. If patients had subjective symptoms or esthetic concerns, surgery was performed by an otolaryngologist, neurosurgeon, plastic surgeon, or as a team effort depending on the location of the tumor. An endoscopic approach was preferred for paranasal BFOL, whereas some patients underwent surgery via an external approach or craniotomy. If there were no symptoms, follow-up imaging was performed at various intervals. If the patient underwent surgery, follow-up imaging was performed between 6 and 12 months after surgery.

\section{Statistical analysis}

Data were presented as mean and standard deviation and tested for normality of distribution using the Shapiro-Wilk test. Comparisons between the surgery and observation groups were performed using the Student $t$-test or the Mann-Whitney $U$-test. The Chi-square test or Fisher's exact test was used for analyzing categorical variables. The Kruskal-Wallis test with Dunn's multiple comparison test was used. Statistical significance was accepted at $P<0.05$. Stata software ver. 14.0 (Stata Corp., College Station, TX, USA) was used. 


\section{RESULTS}

Among a total of 606,068 radiologic evaluations of the paranasal sinuses over 22 years, 183 cases were diagnosed with BFOL

Table 1. Demographics of patients with benign fibro-osseous tumors in the paranasal sinuses

\begin{tabular}{|c|c|c|c|c|}
\hline Characteristics & $\begin{array}{c}\text { Total } \\
(n=183)\end{array}$ & $\begin{array}{l}\text { Surgery } \\
(n=78)\end{array}$ & $\begin{array}{l}\text { Observation } \\
(n=105)\end{array}$ & $P$-value \\
\hline Male sex & $103(56.3)$ & 58 (55.2) & $45(57.7)$ & 0.765 \\
\hline Age at diagnosis (yr) & $28.6 \pm 18.1$ & $21.8 \pm 13.6$ & $33.6 \pm 19.4$ & $<0.001^{\mathrm{a})}$ \\
\hline Follow-up period (mo) & $54.3 \pm 50.2$ & $66.2 \pm 53.3$ & $39.1 \pm 41.6$ & $<0.001^{\text {a) }}$ \\
\hline Reason for imaging & & & & $<0.001^{\text {a) }}$ \\
\hline Diagnostic & $113(61.7)$ & 70 (61.9) & $43(38.1)$ & \\
\hline Incidental & $70(38.3)$ & $8(11.4)$ & $62(88.6)$ & \\
\hline \multicolumn{5}{|l|}{ Sinus involvement } \\
\hline Maxilla & $56(30.6)$ & $26(33.3)$ & $30(28.6)$ & 0.519 \\
\hline Ethmoid & $53(29.0)$ & $31(39.7)$ & $22(21.0)$ & $0.008^{a)}$ \\
\hline Frontal & 95 (51.9) & $44(56.4)$ & $51(48.6)$ & 0.300 \\
\hline Sphenoid & $89(48.6)$ & $40(51.3)$ & $49(46.7)$ & 0.553 \\
\hline $\begin{array}{l}\text { Multiple adjacent bone } \\
\text { involvement }\end{array}$ & $83(45.4)$ & $44(56.4)$ & $39(37.1)$ & $0.010^{a)}$ \\
\hline Skeletal involvement & $13(7.1)$ & $4(5.1)$ & $9(8.6)$ & 0.562 \\
\hline $\begin{array}{l}\text { McCune-Albright } \\
\text { syndrome }\end{array}$ & $7(3.8)$ & $1(1.3)$ & $6(5.7)$ & 0.241 \\
\hline
\end{tabular}

Values are presented as number (\%) or mean \pm standard deviation. a) Statistically significant using the Mann-Whitney U-test. of the paranasal sinuses. The estimated prevalence based on radiology was $0.03 \%$ across age groups. The demographic data of patients in this study are presented in Table 1 . The patients included 103 males $(56.3 \%)$ and 80 females $(43.7 \%)$, ranging in

Table 2. Reason for radiologic imaging studies in patients with benign fibro-osseous lesions

\begin{tabular}{llr}
\hline Category & \multicolumn{1}{c}{ Reason } & No. (\%) \\
\hline Diagnostic & & $113(61.7)$ \\
\cline { 2 - 3 } & Facial asymmetry & $52(46.0)$ \\
& Headache & $12(10.6)$ \\
& Skull mass & $10(8.8)$ \\
& Nose symptom & $10(8.8)$ \\
& Visual loss & $8(7.1)$ \\
& Limb pain or multiple fracture & $7(6.2)$ \\
& Obliteration of external auditory canal & $5(4.4)$ \\
& Others (parotid swelling, meningitis, loss of & $9(8.0)$ \\
& consciousness, skin lesion, facial pain, etc.) & \\
Incidental & $70(38.3)$ \\
& Trauma & $33(47.1)$ \\
& Routine check-up & $10(14.3)$ \\
& Ear symptom (hearing loss or otorrhea) & $6(8.6)$ \\
& Dizziness & $5(7.1)$ \\
& Dental problem & $5(7.1)$ \\
& Others (epistaxis, facial nerve palsy, hemifacial & $11(15.7)$ \\
& spasm, trigeminal neuralgia, seizure, panic & \\
& attack, loss of consciousness, etc.) & \\
\hline
\end{tabular}
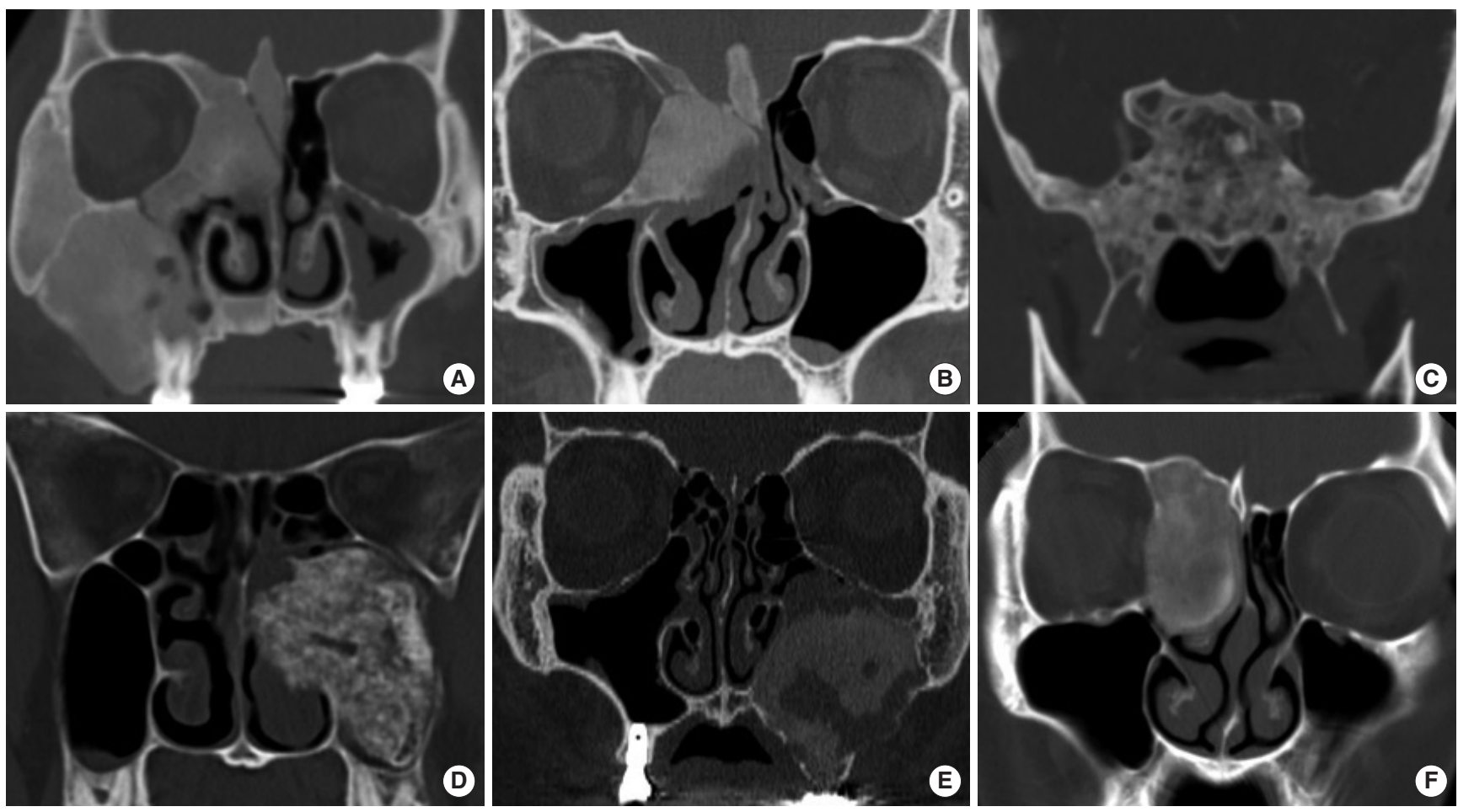

Fig. 1. Radiologic findings of pathologically confirmed benign fibro-osseous lesions of the paranasal sinuses. Computed tomography scans of three different patients with fibrous dysplasia (FD) and ossifying fibroma (OF). (A) FD involving the right maxilla and zygoma. (B) FD confined to the right ethmoid bone. (C) FD of the sphenoid bone. (D, E) OF in the left maxillary sinus. (F) OF in the right ethmoid sinus. 
age from 6 months to 79.5 years old (mean age, 28.6 \pm 18.1 years). The reasons for imaging evaluation were divided into three categories. The majority of patients $(113 / 183,61.7 \%)$ visited because of specific symptoms related to BFOLs, such as facial asymmetry, headache, skull mass, ear, and nasal symptoms, or visual loss. Seventy patients (38.3\%) had no signs, but were incidentally diagnosed based on radiologic imaging for trauma, routine health check-ups, or dental problems. Seventy-eight patients $(42.6 \%)$ underwent surgical treatment. When the surgery group and observation group were compared (Table 1), the patients who underwent surgery were, on average, younger $(P<0.001)$ and had a longer follow-up duration $(P<0.001)$. The observation group was more frequently diagnosed incidentally $(P<0.001)$.

The specific reasons for imaging are summarized in Table 2. Facial asymmetry was the most common reason for diagnostic imaging, and trauma was the most common precursor of incidental detection. The involved sinuses and ranges varied, and seven patients had McCune-Albright syndrome. The radiologic findings of FD and OF confirmed on pathology are illustrated in Fig. 1. The radiologic differential diagnosis of FD and OF included osteoma, osteoblastoma, osteosarcoma, chondrosarcoma, meningioma, odontoma, metastasis, eosinophilic granuloma, or intraosseous hemangioma. The most common pathologic finding was FD. Other surgical pathology findings were OF, osteoma, osteoblastoma, periapical cyst, and spindle cell tumor (Table 3).

Table 3. Surgical pathology results of patients who underwent surgery or biopsy according to an initial radiologic diagnosis

\begin{tabular}{lc}
\hline Variable & No. $(\%)$ \\
\hline Fibrous dysplasia $(\mathrm{n}=73)$ & \\
\hline Fibrous dysplasia & $50(68.5)$ \\
Ossifying fibroma & $3(4.1)$ \\
Osteoblastoma & $1(1.4)$ \\
Spindle cell tumor & $1(1.4)$ \\
Nondiagnostic & $6(8.2)$ \\
No report & $12(16.4)$ \\
Ossifying fibroma $(\mathrm{n}=14)$ & \\
Fibrous dysplasia & $5(35.7)$ \\
Ossifying fibroma & $4(28.6)$ \\
Osteoma & $1(7.1)$ \\
Osteoblastoma & $1(7.1)$ \\
Periapical cyst & $2(14.3)$ \\
Spindle cell tumor & $1(7.1)$ \\
\hline
\end{tabular}
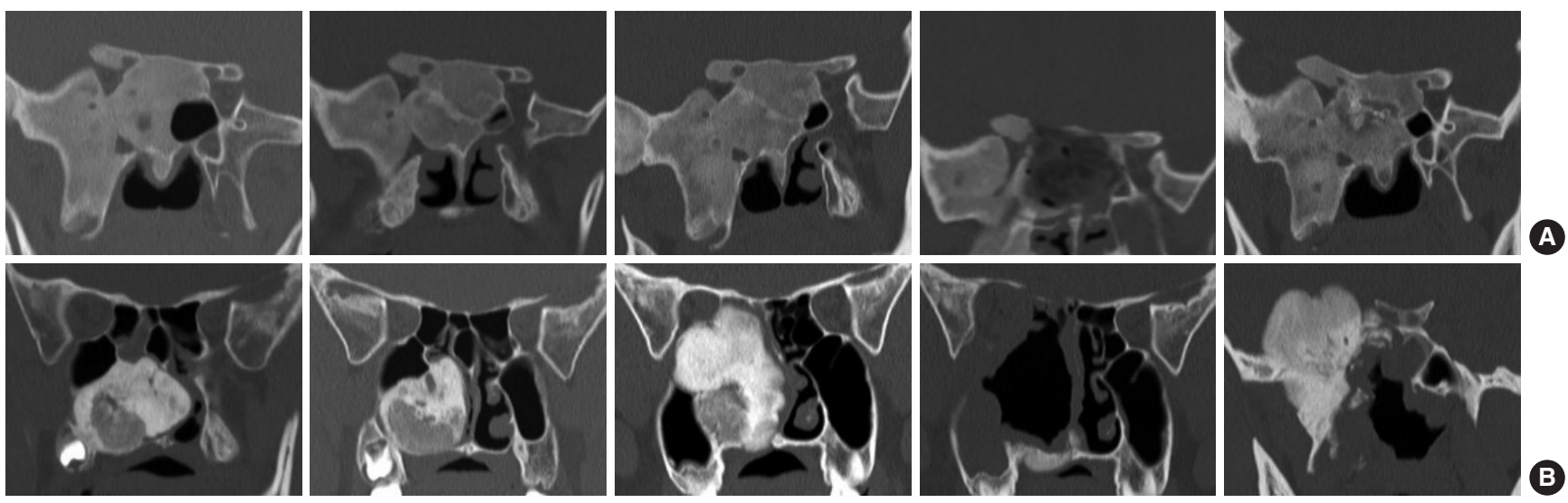

Fig. 2. Cases with tumor regrowth after multiple operations. (A) An 8-year-old female patient with fibrous dysplasia who underwent four operations. (B) A 12-year-old female patient experienced a malignant transformation of ossifying fibroma to osteosarcoma.

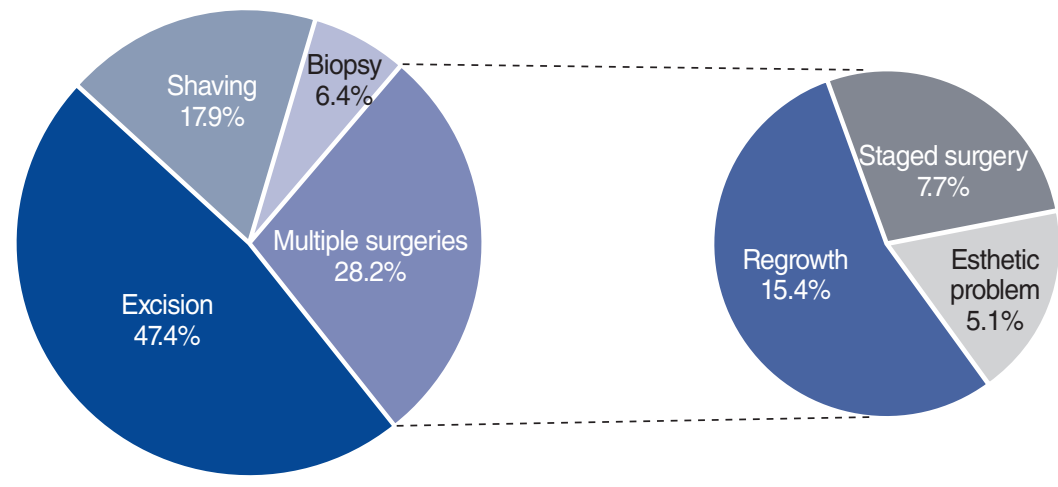

Fig. 3. The reasons for surgery in patients with benign fibro-osseous lesions. 
Table 4. Characteristics of patients according to size change of benign fibro-osseous tumors in the paranasal sinuses

\begin{tabular}{|c|c|c|c|c|c|}
\hline \multirow[b]{2}{*}{ Variable } & \multicolumn{2}{|c|}{ Observation group } & \multicolumn{2}{|c|}{ Surgery group } & \multirow[b]{2}{*}{$P$-value } \\
\hline & No change & Increased size & $\begin{array}{l}\text { No change after } \\
\text { surgery }\end{array}$ & $\begin{array}{c}\text { Increased size after } \\
\text { surgery }\end{array}$ & \\
\hline Number & 49 & 7 & 66 & 8 & \\
\hline Age at diagnosis (yr) & $31.6 \pm 18.3^{a)}$ & $10.9 \pm 8.6^{a), b)}$ & $21.8 \pm 13.2^{b)}$ & $17.7 \pm 15.3$ & $0.001^{c)}$ \\
\hline Follow-up period (mo) & $4.9 \pm 41.0^{\mathrm{a}), \mathrm{b})}$ & $65.2 \pm 31.6$ & $66.8 \pm 56.5^{\mathrm{a})}$ & $73.5 \pm 31.3^{b)}$ & $<0.001^{c)}$ \\
\hline Multiple adjacent bone involvement & $26(53.1)$ & $4(57.1)$ & $34(51.5)$ & $6(75.0)$ & 0.694 \\
\hline Sites of involvement & $1.9 \pm 1.3$ & $2.4 \pm 1.4$ & $2.1 \pm 1.5$ & $3.3 \pm 1.9$ & 0.237 \\
\hline Histologic diagnosis ${ }^{\mathrm{d})}$ & & & & & - \\
\hline Fibrous dysplasia & $2(50.0)$ & & $42(63.6)$ & $6(75)$ & \\
\hline Ossifying fibroma & $1(25.0)$ & & $4(6.1)$ & & \\
\hline Osteoblastoma & & & & $1(12.5)$ & \\
\hline Osteoma & & & $1(1.5)$ & & \\
\hline Spindle cell tumor & & & & $1(12.5)$ & \\
\hline Periapical cyst & & & $2(3.0)$ & & \\
\hline Nondiagnostic & $1(25.0)$ & & $5(7.6)$ & & \\
\hline No report & & & $12(18.2)$ & & \\
\hline Visual symptom & 1 & & 5 & 3 & - \\
\hline Skeletal involvement & 3 & 3 & 3 & 1 & - \\
\hline McCune-Albright syndrome & 2 & 2 & & 1 & - \\
\hline
\end{tabular}

Values are presented as mean \pm standard deviation or number (\%).

a).b) Indicated statistically statistically significant $(P<0.05)$ between two groups marked with same footnotes by the Dunn's multiple comparison test. ${ }^{c)}$ Statistically significant using Kruskal-Wallis test with Dunn's multiple comparison test. ${ }^{d)}$ Four patients underwent only endoscopic biopsy.
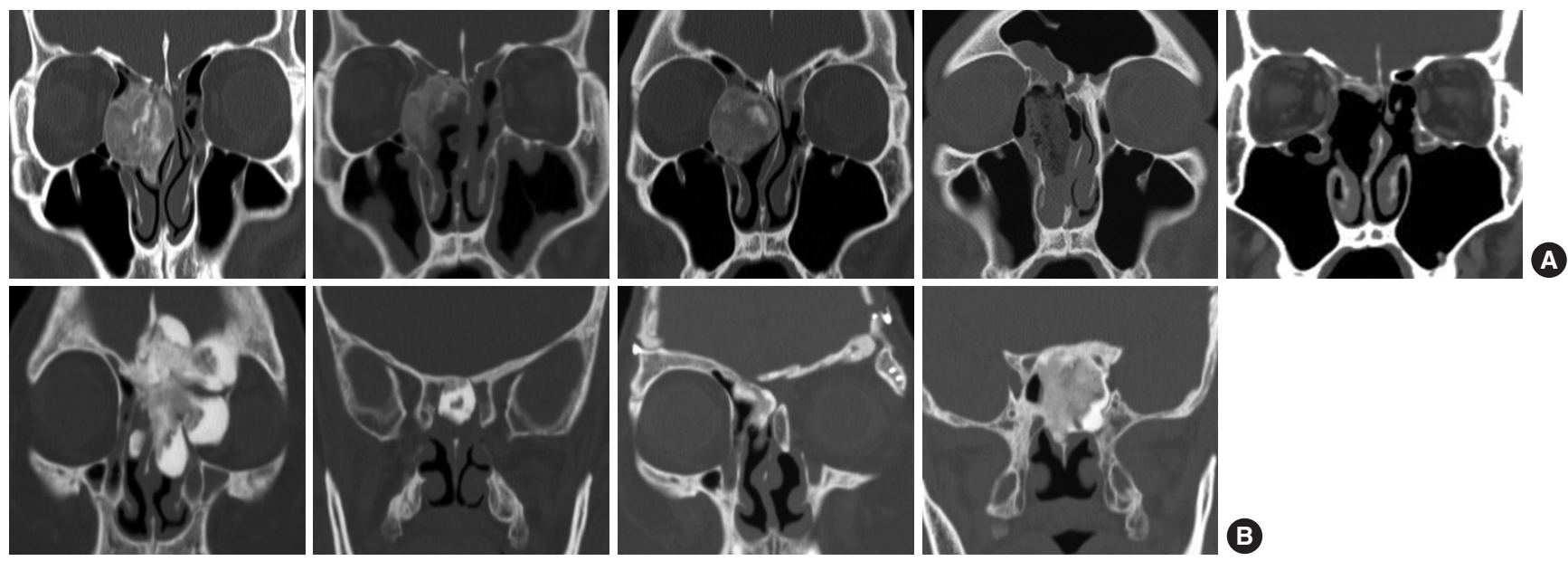

Fig. 4. Adult cases with lesion growth after surgery. (A) A 23-year-old male patient with ossifying fibroma in the right ethmoid sinus underwent surgery twice due to tumor regrowth after partial resection. Computed tomography (CT) images of initial, first postoperative, recurrent, second postoperative (with packing material), and final status. (B) A 53-year-old female patient with fibrous dysplasia exhibited lesion growth in the sphenoid sinus after surgery, as shown on two preoperative and two postoperative CT images.

In the surgery group, 16 patients $(20.5 \%)$ underwent two operations, three patients underwent three operations, and three patients underwent four operations. One of the FD patients who underwent four operations showed repeated tumor regrowth in the sphenoid sinus (Fig. 2A). Overall, 22 of 78 patients (28.2\%) underwent two or more operations. The reasons for surgery were excision $(47.4 \%)$, shaving $(17.9 \%)$, and biopsy $(6.4 \%)$ in patients who underwent surgery once (Fig. 3). The reason for multiple surgical procedures was tumor regrowth in 12 cases, staged operations in 6 cases, and esthetic problems in four cases. According to the pathology reports, 18 patients had FD and three patients had OF.

Among the 130 patients with follow-up information (defined as patients with follow-up images at more than a 6-month interval after initial imaging), those who experienced lesion growth $(15 / 130,11.5 \%)$ tended to be younger and to have more sites of involvement (Table 4). Most patients with lesion growth were in their teens, except for three patients $(23,27$, and 58 years 

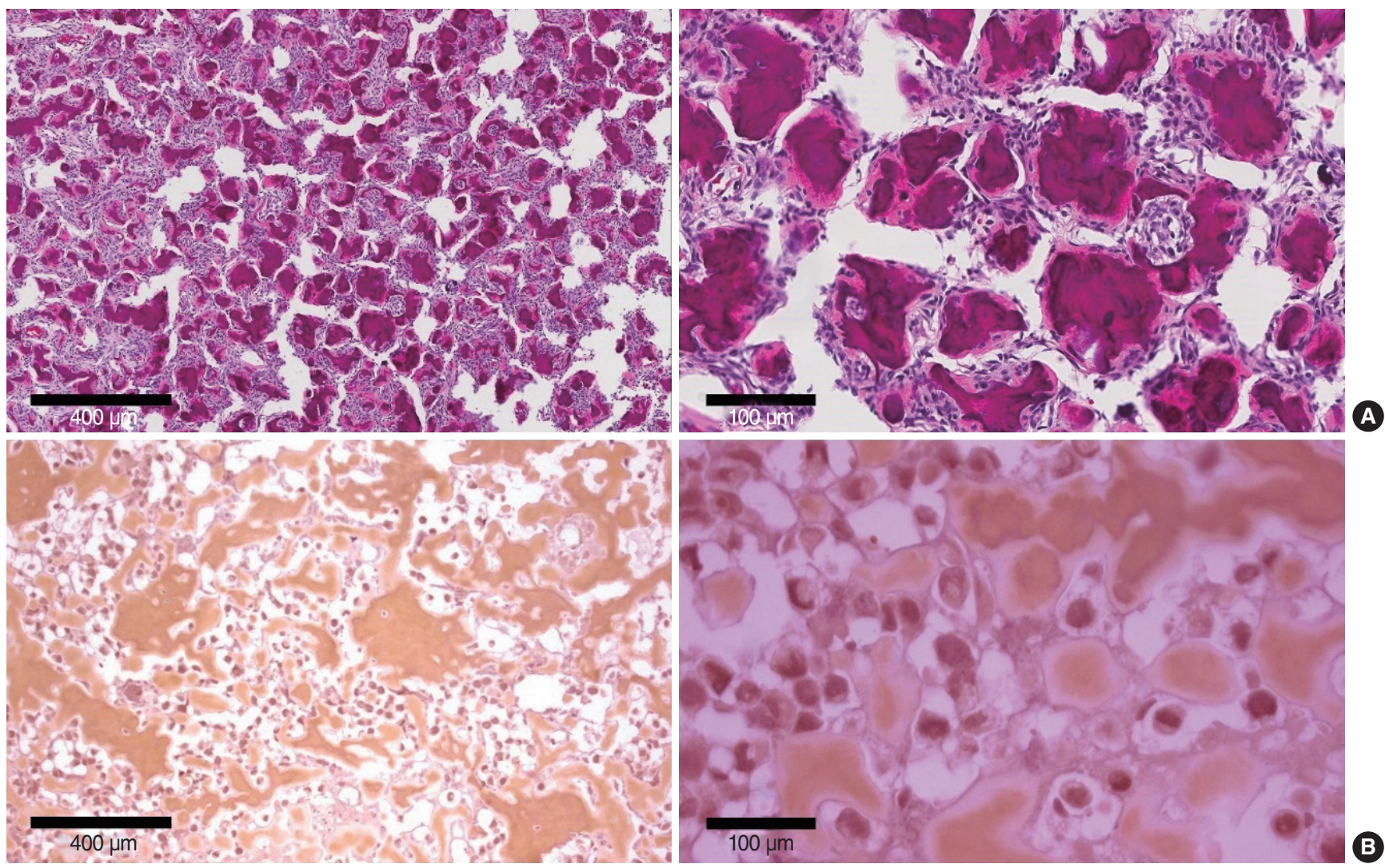

Fig. 5. Histologic findings (H\&E) of a 12-year-old patient with malignant transformation. (A) Ossifying fibroma at the first surgery. Mineralized matrix with stromal fibroblastic cells with innumerable psammomatoid calcifications. (B) Osteosarcoma at the third surgery. Pleomorphic atypical cells of various sizes with large nuclei. Osteoids with a dense and amorphous extracellular matrix.

old). Among the three adult patients, two underwent surgical treatment; one patient was diagnosed with $\mathrm{OF}$ (Fig. 4A) and the other with FD (Fig. 4B). The number of patients with skeletal involvement and McCune-Albright syndrome was not significantly different between patients with no change and those who experienced lesion growth. Some patients had visual symptoms, including diplopia, visual acuity decrement, and visual field defects, and five patients underwent optic nerve decompression surgery. One patient (12 years old) experienced a malignant transformation of $\mathrm{OF}$ and underwent medial maxillectomy with a final diagnosis of osteosarcoma (Figs. 2B and 5).

\section{DISCUSSION}

In this comprehensive review of BFOLs of the sinonasal region, the estimated prevalence was $0.03 \%$. Seventy-eight of the 183 patients $(42.6 \%)$ underwent surgery, and 15 of the 130 patients (11.5\%) experienced tumor growth during the follow-up period regardless of whether they underwent surgery. BFOL growth in the paranasal sinuses was more common in younger patients in their teens with numerous sites of involvement.

A radiologic finding of BFOL alone is not sufficient for an ac- curate diagnosis, and a comprehensive approach is mandatory, including an assessment of the patient's demographic characteristics and clinical features, as well as the tumor's gross appearance and histology [5].The histomorphological patterns of BFOLs are similar among different entities, and some distinct BFOLs may even have identical microscopic features [2]. Efune et al. [6] evaluated the concordance rate between radiology and histology of paranasal fibro-osseous lesions, and positive predictive value of radiologic findings was $100 \%$ for osteoma, $85.7 \%$ for FD, and $33.3 \%$ for OF. Osteoma is the most common BFOL in the paranasal sinuses, and few cases of osteomas generally require surgical treatment. Therefore, our study included FD and OF cases from radiologic studies, with the exclusion of osteomas.

As mentioned in previous studies, the main presentation of BFOL is craniofacial asymmetry or deformity and headache [9]. Our data also showed that 102 of 183 patients (55.7\%) underwent imaging studies because of specific symptoms, such as facial asymmetry, headache, a skull mass, ear or nose discomfort, or visual disturbance. However, a large portion of cases (37.7\%) were detected incidentally (Table 1 ). The majority of patients were diagnosed with FD radiologically and pathologically. The second most common disease entity was OF, but its prevalence was very low. These two diseases occasionally have been con- 
fused in pathologic diagnosis [10,11]. OF requires complete excision because of the possibility of recurrence or malignant transformation $[12,13]$. In our data, a 23-year-old patient with $\mathrm{OF}$ experienced tumor recurrence after partial resection (Fig. 4A). A 12-year-old patient also experienced a malignant transformation from OF to osteosarcoma (Figs. 2B and 5). If the clinico-radiologic manifestations are aggravated, it is essential to perform a biopsy during surgical treatment to monitor for malignant transformation.

However, there are not yet any standard treatments for BFOLs. The recommended treatment for asymptomatic BFOLs is observation with serial radiologic imaging. No definitive medical treatments exist for BFOLs with a curative objective. Medical treatment is based on the anti-resorptive agent pamidronate, which is a second-generation bisphosphonate that reduces bone pain and can lead to the refilling of osteolytic lesions or thickening of cortices [14]. However, a study in children and adolescents found no clear evidence that pamidronate affects dysplastic lesions [15]. Surgical treatment is the most effective treatment modality for BFOLs. Therapeutic planning should depend on the anatomic site and the range of involvement. When surgery is indicated for the relief of symptoms, endoscopic endonasal techniques are suitable for craniofacial lesions with excellent cosmetic outcomes [16]. An endoscopic approach is the most common choice of treatment for craniofacial BFOLs [17]. However, some cases require an external approach due to the location of the tumor. Conservative surgery for FD was recommended by some researchers; specifically, it was suggested that periodic shaving might be helpful until a static phase is reached [18]. Some surgeons prefer radical excision, which necessitates complete removal of the lesion, followed by immediate reconstruction [19].

Considering that the patients who underwent surgery were younger than the patients in the observation group and that younger patients were more likely to experience tumor growth during the follow-up period, growth hormone is suspected to have affected tumor growth. An excess of growth hormone is an established risk factor for compressive optic neuropathy in patients with FD $[20,21]$. Therefore, careful and regular follow-up is required for younger patients in their teens. Surgeons should be cautious of tumor regrowth, complications, or malignant transformation in teenaged patients with BFOL.

There are some limitations to this study. First, because it is a retrospective study based on medical records, it may have been affected by selection bias. In our study, 78 patients ( $42.6 \%$ ) underwent surgery because of subjective symptoms or esthetic concerns, and the others belonged to the observation group. However, the observation group was not matched to the control group in terms of disease extent or severity. It is possible that the surgery group included patients with more severe and extended diseases. In particular, the decision of whether to perform surgery may depend on the preference of the surgeon. In the observation group, some patients were lost to follow-up, so we could not identify whether they underwent surgery or the lesion grew. Second, surgical procedures were not evaluated in this study in detail due to the nature of this retrospective study. Third, biopsy results from 10 of 78 patients who underwent surgery proved to be nondiagnostic or unreported. Histologic confirmation should be performed, even if the diagnosis is made by radiologic imaging. Despite these limitations, to our knowledge, this is the largest single-center study to date, comprising 183 BFOLs of the paranasal sinuses, mainly including FD and OF. Our efforts to describe the clinical course of BFOLs will inform future treatment strategies.

\section{CONFLICT OF INTEREST}

No potential conflict of interest relevant to this article was reported.

\section{ORCID}

Jung Joo Lee

Gwanghui Ryu

Kyung Eun Lee

Sang Duk Hong

Yong Gi Jung

Hyo Yeol Kim

Hun-Jong Dhong

Seung-Kyu Chung

https://orcid.org/0000-0002-8078-345X

https://orcid.org/0000-0002-3251-399X

https://orcid.org/0000-0001-8248-2020

https://orcid.org/0000-0003-3075-1035

https://orcid.org/0000-0001-7456-849X

https://orcid.org/0000-0002-2162-3202

https://orcid.org/0000-0003-0054-9629

https://orcid.org/0000-0001-6187-0980

\section{AUTHOR CONTRIBUTIONS}

Conceptualization: JJL, GR, SDH, HJD, SKC. Formal analysis: JJL, GR, KEL. Methodology: SDH, YGJ, HYK. Visualization \& Writing-original draft: JJL, GR. Writing-review \& editing: SDH, HJD, SKC.

\section{REFERENCES}

1. Waldron CA, Giansanti JS. Benign fibro-osseous lesions of the jaws: a clinical-radiologic-histologic review of sixty-five cases. Oral Surg Oral Med Oral Pathol. 1973 Feb;35(2):190-201.

2. Eversole R, Su L, ElMofty S. Benign fibro-osseous lesions of the craniofacial complex: a review. Head Neck Pathol. 2008 Sep;2(3):177202.

3. El-Mofty SK. Fibro-osseous lesions of the craniofacial skeleton: an update. Head Neck Pathol. 2014 Dec;8(4):432-44.

4. Godt A, Gulicher D, Kalwitzki M, Krober SM. Dislocation of an upper third molar by an ossifying fibroma: case report. J Craniomaxillofac Surg. 2008 Sep;36(6):360-4.

5. Nelson BL, Phillips BJ. Benign fibro-osseous lesions of the head and neck. Head Neck Pathol. 2019 Sep;13(3):466-75. 
6. Efune G, Perez CL, Tong L, Rihani J, Batra PS. Paranasal sinus and skull base fibro-osseous lesions: when is biopsy indicated for diagnosis? Int Forum Allergy Rhinol. 2012 Mar-Apr;2(2):160-5.

7. Zeng HF, Lu JJ,Teng L, Jin XL, Xu JJ, Zhang C, et al. Surgical treatment of craniomaxillofacial fibrous dysplasia: functionally or aesthetically? J Craniofac Surg. 2013 May;24(3):758-62.

8. Hocaoglu E, Yirgin IK, Aksoy S, Arslan G, Inci E, CimilliT. Computed tomography imaging findings of craniofacial fibrous dysplasia. J Craniofac Surg. 2014 Jul;25(4):1175-7.

9. Fadle KN, Hassanein AG, Kasim AK. Orbitocranial fibrous dysplasia: outcome of radical resection and immediate reconstruction with titanium mesh and pericranial flap. J Craniofac Surg. 2016 Nov;27(8): e719-23.

10. Turin SY, Purnell C, Gosain AK. Fibrous dysplasia and juvenile psammomatoid ossifying fibroma: a case of mistaken identity. Cleft Palate Craniofac J. 2019 Sep;56(8):1083-8.

11. Veltrini VC, Figueira JA, Santin GC, de Sousa SC, de Araujo NS. Can non-collagenous proteins be employed for the differential diagnosis among fibrous dysplasia, cemento-osseous dysplasia and cemento-ossifying fibroma? Pathol Res Pract. 2019 Jul;215(7):152450.

12. Liu Y,Wang H, You M, Yang Z, Miao J, Shimizutani K, et al. Ossifying fibromas of the jaw bone: 20 cases. Dentomaxillofac Radiol. 2010 Jan;39(1):57-63.

13. Wang K, Ma XJ, Hao SY, Du J, Zhang LW, Zhang JT, et al. Skull base juvenile psammomatoid ossifying fibroma: clinical characteristics, treatment, and prognosis. World Neurosurg. 2019 May;125:e843-8.
14. Liens D, Delmas PD, Meunier PJ. Long-term effects of intravenous pamidronate in fibrous dysplasia of bone. Lancet. 1994Apr;343(8903): 953-4.

15. Plotkin H, Rauch F, Zeitlin L, Munns C, Travers R, Glorieux FH. Effect of pamidronate treatment in children with polyostotic fibrous dysplasia of bone. J Clin Endocrinol Metab. 2003 Oct;88(10):4569-75.

16. Duan C, Dai Q, Liu Q, Yu H. Characteristics of sinonasal fibrous dysplasia: experience from a single department. Acta Otolaryngol. 2018 Jan;138(1):50-5.

17. Ciniglio Appiani M, Verillaud B, Bresson D, Sauvaget E, Blancal JP, Guichard JP, et al. Ossifying fibromas of the paranasal sinuses: diagnosis and management. Acta Otorhinolaryngol Ital. 2015 Oct;35(5): 355-61.

18. Ramsey HE, Strong EW, Frazell EL. Fibrous dysplasia of the craniofacial bones. Am J Surg. 1968 Oct;116(4):542-7.

19. Zenn MR, Zuniga J. Treatment of fibrous dysplasia of the mandible with radical excision and immediate reconstruction: case report. J Craniofac Surg. 2001 May;12(3):259-63.

20. Lee JS, FitzGibbon E, Butman JA, Dufresne CR, Kushner H, Wientroub $\mathrm{S}$, et al. Normal vision despite narrowing of the optic canal in fibrous dysplasia. N Engl J Med. 2002 Nov;347(21):1670-6.

21. Cutler CM, Lee JS, Butman JA, FitzGibbon EJ, Kelly MH, Brillante BA, et al. Long-term outcome of optic nerve encasement and optic nerve decompression in patients with fibrous dysplasia: risk factors for blindness and safety of observation. Neurosurgery. 2006 Nov; 59(5):1011-7. 